\title{
Rhétorique et techniques de persuasion chez le général de Gaulle (Discours du 4 septembre 1958)
}

\section{Dr. Rania Ezz El Arab}

Maître de conferénces, Département de langue et de littératures françaises, Faculté des lettres

Université d'Alexandrie 
Le 4 septembre 1958, jour anniversaire de la proclamation de la République en 1870, le général de Gaulle présenta au peuple français le projet de Constitution que le gouvernement venait d'adopter, et qui allait être soumis au référendum du 28 septembre.

Par son discours, le général considère une cible précise: sa rhétorique doit convaincre essentiellement et dans l'immédiat le peuple français de voter "oui" à la constitution. Ainsi apparaît la problématique: le maniement de la rhétorique par un homme qui défend un point de vue qui n'est pas nécessairement celui des autres. Mais l'homme en question n'est pas un homme ordinaire. C'est un chef charismatique qui occupe une place importante aux yeux du monde et évidemment de la France. De même, la cause qu'il défend est une grande cause: établir une nouvelle constitution donc restaurer une nouvelle république (la Vème). C'est l'avenir de la France et de son chef qui sont en jeu, et c'est précisément la rhétorique ou plutôt "sa" rhétorique qui décidera du sort du général et de celui de la France. La situation ne permet aucun échec.

La rhétorique est donc une arme redoutable. Tout comme l'épée, elle n'offre à l'utilisateur que deux possibilités: une sortie triomphale du champ de bataille et donc une victoire décisive-si celui-ci arrive à convaincre le récepteur de son point de vue; sinon une défaite humiliante et sans lendemain si l'émetteur manque d'habileté et qu'il utilise 
maladroitement l'arme qui lui est offerte. Tout comme sur le champ de bataille, si l'émetteur est battu, jamais il n'aura l'occasion de recommencer. Il perd sa crédibilité. Cette image est loin d'être une métaphore. Si la rhétorique trouve ses racines en Sicile, avant de devenir un art ou une science controversée, dans l'Athènes du Vème siècle avant $\mathrm{JC}$, elle doit son succès, comme l'a savamment démontré Françoise BOURSIN «à son pouvoir ou plutôt au pouvoir qu'elle offre à ceux qui veulent utiliser la parole ${ }^{1} »$. Au temps de Périclès, enseignée par les grands sophistes comme Protagoras ou Gorgias, elle était aussi utilisée et admirée que les armes de la guerre; et les échanges verbaux étaient aussi redoutables que les fils de l'épée. L'agora, où se déroulaient à la fois les grandes batailles et les éminentes discussions philosophiques, en est témoin. Mais quelle est cette aura qui entourait la rhétorique? Pourquoi cette place lui était-elle réservée? Parce qu' «elle servait à convaincre et donc à obtenir l'adhésion pour gouverner, dans un monde où la politique et le juridique étaient souverains ${ }^{2} »$.

Toutefois, la rhétorique revêt deux aspects: l'aspect positif lié à l'atmosphère même qui, elle, favorise ou non l'épanouissement de la rhétorique car cette dernière ne peut fonctionner qu'à deux conditions: dans un monde de liberté,

\footnotetext{
1 BOURSIN Françoise, Dominique de Villepin et Colin Powell: deux rhétoriques face à face, in Communication et langages, $2005, \mathbf{n}^{\circ} 145$, pp.95106.

${ }^{2}$ Ibid, loc.cit.
} 
de démocratie, loin de toute contrainte autoritaire, d'une part; dans un monde où l'orateur a la possibilité matérielle de toucher le récepteur, d'autre part. C'est cette utilisation positive de la rhétorique que peint d'une manière si vivante Jacqueline de Romilly ${ }^{3}$, en racontant les joutes oratoires de l'Athènes antique avec une modernité saisissante.

Quant à l'aspect négatif, il relève du fait que la rhétorique s'appuie sur le vraisemblable et non sur le vrai, et se soucie donc assez peu de la vérité. Autrement dit, elle ne recherche que l'efficacité, sans scrupule éthique ce qui l'apparente fortement à la manipulation. C'est la vision qu'ont largement transmise à la postérité, Socrate et son célèbre disciple Platon. Malgré une conception plus objective d'Aristote, qui ne la considère que comme un outil, utilisé à bon ou à mauvais escient et plus optimiste de Cicéron, qui manifeste dans son jugement de praticien, de théoricien et de penseur, son éternelle confiance dans l'humanitas, elle reste longtemps entachée de soupçons, et n'échappe pas à des voisinages compromettants tels que ceux de Machiavel ou des propagandes totalitaires.

La rhétorique a traversé vingt cinq siècles, avec plus ou moins de bonheur, de succès ou de rejet. Mais appréciée depuis fort longtemps aux Etats-Unis, elle est peu à peu réhabilitée en France. Elle semble retrouver plus ou moins

3 Jacqueline de Romilly, Les grands Sophistes dans l'Athènes de Périclès, "Biblio Essais", Editions de Fallois, Paris, 1988, p.287. 
sa gloire d'antan à travers les discours politiques français, surtout lorsque leurs émetteurs sont «cultivés» et précisément renommés pour leur culture classique. Dans ce cas précis, ces émetteurs, à l'instar des sophistes, mettent la rhétorique au service de leur objectif et de leur ambition: convaincre le monde de suivre leur choix.

C'est le cas d'un grand nombre de diplomates français dont, à titre d'exemple, Dominique de Villepin, ministre des affaires étrangères sous le mandat de Jacques Chirac qui lui, est renommé d'avoir été le disciple de l'un des grands maîtres de la grandiloquence- dans le bon sens du terme - à savoir le général de Gaulle.

La méthodologie que nous proposons reposera donc sur un seul axe, celui de la rhétorique, autrement dit l'art de bien parler,-l'art de la parole publique efficace selon la définition des Grecs-, la technique de la mise en œuvre des moyens d'expression. Cependant, cette étude des moyens d'expression est loin d'être un travail de recensement: en fait, de ces moyens d'expression, nous n'avons choisi que ceux qui nous ont paru les plus importants, les plus révélateurs.

Comme nous l'avons déjà mentionné, l'objectif du discours du général de Gaulle est bien déterminé: remporter l'adhésion des Français à la nouvelle république. Ceci, dès la première phrase. Pour défendre son point de vue, il utilise des arguments multiples: le rôle indéniable qu'à joué le 
régime républicain en France depuis l'avènement de celui-ci (en reconnaissant précisément le mérite des trois premières Républiques), la bonne intention de la IVème République malgré la faiblesse qu'elle a dû témoigné - et les efforts déployés pour la sauver (en énumérant les démarches prises pour ce faire), l'incapacité du régime actuel (la IVème République) devant les défis grandissants (intérieurs et extérieurs) et l'urgence d'établir une nouvelle république dotée d'une nouvelle constitution. Cette nouvelle république sera, sans aucun doute, à la hauteur de la tâche qui lui est assignée (ses qualités sont énumérées dans le discours), puisqu'elle tiendra compte des défauts de la république «précédente», défauts qu'elle comblera à travers la création d'une nouvelle constitution. Ces arguments sont liés les uns aux autres, ils provoquent un effet de convergence pour un but unique: voter «oui» à la Constitution et donc à l'avènement de la Vème République.

Cette responsabilité est celle des Français, et de Gaulle leur demande de l'assumer. Il le fait implicitement d'abord, tout au long du discours, en leur énumérant les bienfaits de la nouvelle constitution: (c'est dans un premier temps donc que le texte sera argumentatif), explicitement ensuite lorsque sa demande sera direct: "De tout mon cour, au nom de la France: je vous demande de répondre: OUI!" (c'est dans un

\footnotetext{
${ }^{4}$ Charles de Gaulle, Discours et Messages, T.III, avec le renouveau 19581962, Plon, Paris, 1970.
} 
second temps donc, juste avant la péroraison que le texte sera injonctif ${ }^{5}$ ).

Les ressorts sur lesquels s'appuie le général sont la clairvoyance (puisqu'il voit le mérite des trois premières républiques aussi bien que les faiblesses de la IVème), la lucidité et la vision (pour lui l'avenir est clair, il ne reste que le réaliser), l'honnêteté (il reconnaît l'impuissance de la IVème république à surmonter les obstacles qu'elle est en train de rencontrer) l'esprit critique et la capacité de diagnostiquer (puisqu'il connaît parfaitement les vices de la IVème république et propose la manière d'y remédier).

De premier abord, le thème qu'il traite et les arguments qu'il avance sont assez clairs. Mais la tâche la plus difficile et la plus importante est celle de mener à bien la rhétorique, ce qui implique le recours à des techniques de persuasion qui soient capables de mettre d'abord en relief l'idée principale du texte pour parvenir ensuite à orienter le vote pour la constitution.

\footnotetext{
${ }^{5}$ L'étude de la nature du texte ne fait pas partie de notre étude,nous n'allons donc pas nous attarder sur ce point, ceci n'empêche guère de donner la définition du texte argumentatif et de celle du texte injonctif. Le premier, "texte d'idées, a pour fonction de réfuter une thèse adverse et de convaincre. Il utilise des arguments et toute une rhétorique de la persuasion et de la disposition. Les discours préparés, les essais, la littérature engagée, certains articles de presse et textes scientifiques sont de nature argumentative". Le deuxième, le texte injonctif, "a pour but de conseiller et de mobiliser ses lecteurs. Il incite à penser puis à s'engager. Plus il est passionnel et plus il affirme un style comme technique de persuasion."C. Peyroutet, Style et rhétorique, Nathan, Paris, 1998, p.108 -110.
} 
Vue cette situation d'emphase où il est impératif de remporter l'adhésion, techniques de persuasion et figures de rhétorique abondent. Les structures des phrases en sont témoins.

$\mathrm{Ni}$ simple (type sujet + verbe + complément) ni composée,(assemblage de phrases simples juxtaposées et coordonnées) la première phrase est une phrase complexe où les soulignements ${ }^{6}$ sont évidents, les déplacements emphatiques. "C'est en un temps où il fallait se réformer ou se briser que notre peuple, pour la première fois, recourut à la République."

Le groupe sujet (notre peuple) n'est pas uniquement précédé par un groupe circonstanciel assez long (c'est en un temps ou il fallait se réformer ou se briser) mais est séparé en plus de son verbe (recourut) par un autre groupe circonstanciel plus ou moins long (pour la première fois). Le déplacement, qui est un genre de soulignement et qui est né de la construction c'est...que, permet de privilégier le groupe sujet mis alors au milieu de la phrase et crée, par conséquent, un effet emphatique. Le verbe, situé en fin de phrase, produit un rythme ascendant qui ajoute à l'emphase, attire l'attention du récepteur et témoigne d'une grande beauté du style.

\footnotetext{
${ }^{6}$ SOULIGNEMENT On attire l'attention, par divers moyens, sur certaines parties du texte. Parmi les modes de soulignement: l'emploi de c'est...qui...(tour présentatif ou emphasis).
} 
Les phrases construites sur ce modèle sont nombreuses: ainsi, à la ligne 6 de Gaulle dit: «c'est alors $q u$ 'au milieu de la tourmente nationale et de la guerre étrangère apparut la République». A la ligne 52 à 55: «C'est dans la légalité que moi-même et mon gouvernement avons assumé le mandat exceptionnel d'établir un projet de nouvelle Constitution...». A la ligne 65-66, parlant de la constitution qu'il met entre les mains du peuple il ajoute: «Mais c'est en toute conscience que nous la lui proposons." Il avance, enfin, à la ligne 67-68 «Ce qui, pour les pouvoirs publiques, est désormais primordial, $c$ 'est leur efficacité et leur continuité».

Toutes ces phrases produisent le même effet emphatique et servent à mettre en relief la partie de la phrase que l'émetteur veut souligner. Le choix de celui-ci est fait d'après l'importance qu'il accorde à chaque partie de la phrase. Ceci en vu d'être bien sûr, le plus convaincant possible.

Les déplacements sont parfois plus significatifs, quand l'auteur insiste, sans aucune nécessité grammaticale, à mettre le verbe avant son sujet. Dans ce cas-là, le déplacement, qui ne sert qu'à mettre en relief la proposition principale, devient un véritable procédé de rhétorique. «C'est donc - dit-il - pour le peuple que nous sommes, au siècle et dans le monde où nous sommes, qu'a été établi le projet de Constitution. ${ }^{7}{ }$

\footnotetext{
${ }^{7}$ Discours et Messages, p.44, 1.94-95
} 
Le déplacement du verbe "établir" de la fin de la phrase vers son milieu, témoigne de l'importance qu'il revêt aux yeux du général. Lui et ses collaborateurs ont accompli leur travail, c'est le tour des Français d'accomplir le leur. Incitation indéniable à voter "oui".

Dans cette phrase, le déplacement du verbe toujours de la fin de la phrase vers son milieu aurait pour raison l'obtention d'un effet rythmique, d'un écho sonore: "Mais quand, le 18 juin, commença le combat pour la libération de la France, il fut aussitôt proclamé que la République à refaire serait une République nouvelle. ${ }^{8 "}$ De Gaulle veut certainement mettre côte à côte le verbe "commencer" au passé simple et le mot "combat". Il sait que le rythme joue un rôle primordial dans la conviction surtout dans un texte oratoire.

Il est clair que dans les deux exemples cités ci-dessus, le verbe devance le sujet pour des raisons qui relève de la rhétorique, toute nécessité grammaticale étant exclue.

Plus flagrant encore est l'exemple de déplacement qui figure quelques lignes plus loin. Soulignant ses efforts et ceux de ses collaborateurs dans la préparation de la Constitution, de Gaulle se cite en premier puis cite ensuite son gouvernement. «C'est dans la légalité que moi-même et mon gouvernement avons assumé le mandat exceptionnel

${ }^{8}$ Ibid, p.42, 1.35 
d'établir un projet de nouvelle Constitution" ». Ce faisant, il ne se plie aucunement aux règles de la courtoisie française qui, elles, exigent que celui qui parle se cite en dernier c'est à dire après tous les autres. Néanmoins, une question se pose: de Gaulle, ce personnage dorénavant historique grâce au rôle qu'il a joué comme commandant de la résistance, n'est-il pas maintenant - aux yeux du peuple - le seul garant de la réussite de la République nouvelle? Autrement dit, sa figure de chef charismatique, n'est-elle pas la premièrepour ne pas dire la seule pour laquelle le peuple votera si jamais il vote «oui»? De Gaulle est réaliste, sincère et lucide, il connaît sa valeur en ce moment aux yeux du peuple. Cette valeur, il s'en doutera plus tard, d'où sa démission peu après les événements de mai 1968.

Pourtant, malgré l'abondance des déplacements dans ce discours de la Constitution de 1958, on ne peut pas juger le style du général comme étant pompeux, maniéré, ni même alambiqué. C'est plutôt un style travaillé, recherché puisqu'en fait, dans ce même discours, il existe des phrases composées et même des phrases simples (type: sujet + verbe + COD) où les déplacements sont totalement absents. En voici quelques exemples:

$E x .1$ «a nécessité de rénover l'agriculture et l'industrie, de procurer les moyens de vivre, de travailler, de s'instruire, de se loger, à notre population rajeunie, d'associer les

${ }^{9}$ Ibid., p.42, 1.53 
travailleurs à la marche des entreprises, (groupe sujet) nous pousse (verbe) à être, (............), dynamiques et expéditifs (groupe complément) ${ }^{10}$.

Ex.2 «on a pu sauvegarder la chance ultime de la république $^{11}$ »

$E x .3$ «le rapport entre la métropole et les territoires d'outre-mer exigent une profonde adaptation ${ }^{12}{ }$.

Parfois, un complément circonstanciel est mis au début de la phrase, ce qui n'est pas forcément considéré comme étant un déplacement:

Ex.1 «Cependant (complément circonstanciel), le régime (sujet) comportait (verbe) des vices de fonctionnement (COD) qui avaient pu sembler supportables (subordonnée relative) à une époque assez statique, mais qui n'étaient plus compatibles avec les mouvements humains, les changements économiques, les périls extérieurs, qui précédaient la Deuxième Guerre mondiale ${ }^{13 \text { " }}$.

Un grand nombre de phrases simples existent aussi:

Ex. 2 «Jusqu'alors, au long des siècles (groupe circonstanciel), l'Ancien Régime (sujet) avait réalisé (verbe) l'unité (complément d'objet direct) ... de la France ${ }^{14 \text { ". }}$

\footnotetext{
${ }^{10}$ Ibid.,p.43, 1.77 à 81

${ }^{11}$ Ibid.,p.42,1.51-52

12 Ibid., p.43, 1.86-87

${ }^{13}$ Ibid., p.42, 1.29 à 33

${ }^{14}$ Discours et messages, p.41, 1.3-4
} 
Ex.3 «Certes (complément circonstanciel), la République (sujet) a revêtu (verbe) des formes diverses (COD) au cours de ses règnes successifs (complément circonstanciel) ${ }^{15}$ ».

Cette alternance des phrases simples, composées et complexes introduit la variété et est, dans le cas gaullien, un gage de style. Pourquoi? Parce que cette alternance n'est pas utilisée arbitrairement. Elle est «mesurée». Elle cède souvent le pas à une autre caractéristique, peut-être paradoxale : le parallélisme.

A l'instar des déplacements et de l'alternance des phrases, ce parallélisme qui, comme son nom l'indique, consiste à mettre en parallèle des mots et des structures, fait toujours partie du domaine de la combinaison des mots. Quoique parfois un peu théâtrales, ces divers procédés de parallélisme sont liés à l'emphase, à une certaine grandiloquence à laquelle tient absolument le général.

Le parallélisme est utilisé par le général pour attirer l'attention sur des rapports de similitude ou de différence. Il a aussi pour but de créer des effets très facilement identifiables: régularité, symétrie, naissance d'un rythme binaire (et parfois ternaire). Le parallélisme facilite surtout la compréhension et la lisibilité du message, fait bien demandé puisque la plupart des phrases gaulliennes, qu'elles

\footnotetext{
${ }^{15}$ Ibid., 1.12-13
} 
soient complexes, composées ou même simples sont presque toujours des phrases longues.

Le parallélisme syntaxique est utilisé par le général dès le début de son discours, précisément au moment où celui-ci dresse un bref panorama de l'histoire des quatre républiques précédentes: «En 1792, on la vit révolutionnaire et guerrière (...). En 1848, on la vit s'élever au-dessus des barricades (...). Le 4 septembre 1870, au lendemain de Sedan, on la vit s'offrir au pays pour réparer le désastre. ${ }^{16}$ »

Toutefois, le parallélisme est surtout flagrant dans la deuxième partie du texte, ou de Gaulle énumère les «qualités» de la Constitution à voter. Dans cette partie, et précisément à partir de la ligne 94 , les phrases, construites selon un parallélisme syntaxique se succèdent à la file:

1. Que le pays puisse être (....) dirigé..................................96

2. Qu'il existe, (........), un arbitre national (...) chargé..................97

3. Qu'il existe un gouvernement qui soit fait pour gouverner............103

4. Qu'il existe un parlement destiné à représenter. 106

${ }^{16}$ Ibid., loc.cit. 
5. Que

6. Qu'un conseil économique et social (...) désigné (...) fournisse......114

7. Qu'un comité constitutionnel, dégagé (....) ait qualité............. 118

8. Que l'autorité judiciaire soit assurée .120

9. Qu'entre la nation française et ceux des territoires d'outre-mer soit formé 123

10. Qu'enfin (.....) le gouvernement ait la charge. 140

Parallélisme syntaxique car, du point de vue syntaxe, toutes les phrases sont construites sur le même modèle:

Pronom relatif (Que) + sujet + verbe (au subjonctif).

A ce parallélisme syntaxique s'ajoute souvent un parallélisme morphologique. Résultat: des ressemblances encore plus étroites lient certaines de ces phrases les unes aux autres. Ainsi:

- Les phrases: 2-3-4 sont identiques. Elles suivent un même modèle: 
Pronom relatif (Que) + sujet (il impersonnel) + verbe (exister au subjonctif),

- Les phrases: 6-7-8 se ressemblent aussi entre elles. Le modèle qu'elles suivent est le suivant:

Pronom relatif $($ Que $)+$ sujet au singulier + adjectif + verbe (au subjonctif), Quand un adjectif épithète (dirigé, chargé, destiné, désigné, dégagé), des verbes à l'infinitif (gouverner, représenter) ou les deux (destiné à représenter - phrase 4) sont ajoutés à ces formules «moules», ces adjectifs sont préférés à d'autres parce qu'ils produisent le même son. En ce cas-là, nous avons un parallélisme phonique qui entraîne obligatoirement un parallélisme rythmique: atout en plus pour garantir plus de conviction.

Toutefois, si certaines phrases échappent aux ressemblances étroites, à ces parallélismes particuliers (morphologique, phonique et rythmique) n'obéissant ainsi qu'au parallélisme général (syntaxique) telles que les phrases $1-5-9$ ce n'est point le fait du hasard. Elles sont insérées intentionnellement par l'auteur. La raison est évidente: ces phrases qui encerclent les autres réunis en groupes, étreignent celles-ci tout en les séparant ; leur rôle consiste à sauver, de toute monotonie, l'ensemble de cette partie. Aussi sont-elles différentes et semblent-elles exclues de ce jeu de parallélisme. Dans ces phrases, nous avons: 
- Des sujets différents : le pays : nom défini, singulier (phrase 1)/ gouvernement et parlement : nom sans article, groupe sujet (phrase 5);

- Des verbes phonétiquement différent : puisse (phrase 1) / collaborent (phrase 5);

- Le pronom relatif «qu» est suivi d'un groupe circonstanciel et non pas d'un nom comme dans toutes les autres phrases: Qu'entre la nation française et ceux des territoires d'outre-mer (phrase 9) ...etc.

La répétition est un procédé lui aussi lié en quelque sorte au parallélisme. Ceci du moment que répéter, c'est redire, donc employer plusieurs fois le même élément linguistique, mot, groupe, phrase. La répétition, qui participe à la fonction phatique du langage, facilite la perception du message. Elle est demandée dans les textes oratoires afin de souligner les idées et de les mettre en valeur. Elle permet aussi d'établir des parallélismes entre les mots répétés. Autre conséquence très appréciée par de Gaulle: l'apparition d'un rythme.

En fait, les répétitions sont remarquablement fréquentes chez l'auteur. Toutefois, elles sont là sous des formes déterminées: anaphore, épiphore, assonance, allitération, homéotéleute...Mais la répétition sporadique d'un mot précis tout au long du texte n'est pas fréquente. Autrement 
dit, les mots ne reviennent pas comme des leitmotivs: le répertoire du vocabulaire gaullien étant assez varié.

Néanmoins, deux mots sont remarquablement plus répétés que tous les autres: celui de "république" (12 fois) et l'adjectif "nouveau" et ses dérivés. (7fois). Ce sont effectivement les deux mots clés du discours.

Le mot de "République" est répété trois fois sur douze dans les dix premières lignes du discours qui s'étale sur une longueur de cent soixante lignes! Deux fois sur trois, la république est à l'image du sauveur: "C'est en un temps où il fallait se réformer ou se briser que notre peuple, pour la première fois, recourut à la République. ${ }^{17 " ~ " C ' e s t ~ a l o r s, ~}$ qu'au milieu de la tourmente nationale et de la guerre étrangère, apparut la République. ${ }^{18 " ~ " L a ~ r e ́ p u b l i q u e ~ a ~}$ revêtu des formes diverses au cours de ses règnes successifs. ${ }^{19 "}$

Cet abus dans l'utilisation du mot témoigne de la primauté de la république aux yeux du général, de la place qu'elle occupe dans son esprit aussi bien que dans son cœur. En un mot, elle est l'idole du général. Cet amour sincère du régime républicain est favorable à la rhétorique: Convaincu luimême de l'importance de ce régime (que ce soit par le cœur ou par l'esprit), les mots qu'il choisira reflèteront cette

\footnotetext{
${ }^{17}$ Discours et messages, p.41, l.1-2

${ }^{18}$ Ibid., 1.6 à 8.

${ }^{19}$ Ibid., 1.12-13
} 
pensée (ou ces sentiments), d'où une plus grande facilité à convaincre autrui, à remporter son adhésion ${ }^{20}$.

Lié dans son esprit au mot de "république", l'adjectif "nouveau" et ses dérivés s'avèrent aussi d'une importance particulière. Dans ce discours, ils puisent leur importance non seulement dans le grand nombre que compte ces dérivés mais de leur emploi même. Tout un champ sémantique de cet adjectif ainsi que des temps de conjugaison différents du verbe qui en dérive sont sous nos yeux.

Ce mot de "renouveau" est vraiment cher à l'auteur des Mémoires d'espoir. Il suffit de rappeler que ce discours fait partie du Tome III des Discours et Messages, dont le titre est Vers le renouveau. De Gaulle qui veut tout changer cherche l'adhésion de son peuple. Ceci compte beaucoup pour lui. Ne vouloir rien changer est le pire des vices. (Donc il faut entreprendre une nouvelle démarche, il faut voter "oui"). De Gaulle parle, preuve à l'appui:

L'Ancien Régime fut renversé parce qu'il se montra "hors d'état de s'adapter à un monde nouveau. ${ }^{21 "}$ "De même, selon de Gaulle, si la IIème République s'est effacée c'est «faute d'avoir accordé l'ordre avec l'élan du renouveau. ${ }^{22}{ }^{\star}$

\footnotetext{
${ }^{20}$ Pour plus de détails sur ce point, cf. supra., choix des mots.

${ }^{21}$ Discours et messages, p.41, 1.5

${ }^{22}$ Ibid., p.42, 1.19
} 
Après l'épreuve gigantesque qu'a dû subir la France à savoir son implication dans la Seconde Guerre mondiale et l'occupation de Paris par les Allemands, Françaises et Français cherchaient à commencer une époque nouvelle où tous les chagrins seraient oubliés. De Gaulle en est conscient, il profite donc de l'occasion pour conduire le pays vers de nouveaux horizons. Il compte commencer par la «République nouvelle ${ }^{23}{ }$ qui, pour s'établir, a d'abord besoin d'une «nouvelle Constitution. ${ }^{24}$ » Pendant les quatre mois qui suivront le référendum, le gouvernement mettra en place de "nouvelles institutions" 25 ". La Vème République, une fois restaurée, aura pour premier but de "rénover

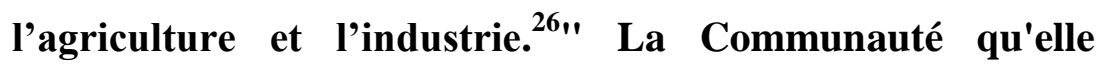
créera "rénovera l'ensemble humain groupé autour de la France. ${ }^{27 "}$

L'usage que de Gaulle fait des répétitions n'est pas toujours sporadique comme le montre les exemples cidessus. Parfois, l'auteur leur confère une place déterminée, une place de premier ordre: en tête de phrase. Appelées ainsi anaphores, ces répétitions produisent un effet qui varie selon le mot utilisé. La première anaphore dans ce discours, de Gaulle y a recours pour parler des difficultés affrontées. C'est ainsi que dans le paragraphe qui commence à la ligne

\footnotetext{
${ }^{23}$ Ibid., 1.37

${ }^{24}$ Ibid., 1.54

${ }^{25}$ Ibid., p.45, 1.144

${ }^{26}$ Ibid., p.43, 1.77

${ }^{27}$ Ibid., p.44, 1.132
} 
39, il met en tête de phrase une expression qu'il répète trois fois en trois lignes à la tête de trois phrases successives :

«On sait, on ne sait que trop, ce qu'il advint de ces espoirs. On sait, on ne sait que trop, qu'une fois le péril passé, tout fut livré et confondu à la discrétion des parties. On sait, on ne sait que trop, quelles en furent les conséquences. ${ }^{28}$ »

Cette manière de «ressasser», loin d'être - comme le prétend Jean - François REVEL «caractéristique de quelqu'un qui a d'abord besoin de se convaincre luimême ${ }^{29}$ " est l'un des procédés de rhétorique auxquels de Gaulle a intentionnellement recours. Dans ce cas précis, l'anaphore, dans un premier temps, aurait pour but de susciter le récepteur à se rappeler lui-même des événements en cause, ce qui sera, sans doute, plus convaincant. Dans un second temps, elle aura pour rôle de nouer entre l'émetteur et le récepteur un lien, nous dirions même une complicité, qui facilitera la compréhension l'un de l'autre.

De Gaulle recourt encore une fois à l'anaphore pour expliquer comment le projet de la Constitution a été élaboré:

«Nous l'avons fait sur la base des principes posés lors de notre investiture. Nous l'avons fait avec la collaboration du Conseil consultatif institué par la loi. Nous l'avons fait compte tenu de l'avis solennel du Conseil d'Etat. Nous

\footnotetext{
${ }^{28}$ Discours et messages, p.42, 1.39 à 42

${ }^{29}$ Revel, Jean-François, Le style du général, Edition René Julliard, Paris,1959, p.92
} 
l'avons fait après délibérations très libres et très approfondies de nos propres Conseils de ministres... ${ }^{30 "}$

Cette fois-ci, l'anaphore a pour but de confirmer l'idée que la Constitution a été préparée très consciencieusement. Le général veut montrer l'effort qui a été déployé en vue de préparer la Constitution de 1958 , il insiste donc sur la qualité de travail qui a donné naissance à cette Constitution: manœuvre assez importante pour rassurer l'auditeur appelé à voter "oui" à cette nouvelle Constitution ${ }^{31}$. Ceci dit, on doit souligner aussi l'utilisation du sujet «nous» qui, loin d'être un «nous» de modestie, est un nous englobant (lui et les Français), un nous qui désigne le pluriel. De Gaulle, une fois de plus, insiste sur le fait que ce travail a été fait dans la démocratie: c'est l'œuvre d'un ensemble réuni de personnes qualifiées et non pas celui d'un seul individu - quelque soit la crédibilité de ce dernier. Ce travail, du moment qu'il est collectif, rehausse certainement la valeur de la nouvelle Constitution surtout quand le récepteur apprend que celle-ci a été élaborée après «des délibérations très libres et très approfondies ${ }^{32}{ }$ et que les Conseils de ministres impliqués dans ce travail sont «formés d'hommes aussi divers que possible d'origines et de tendances, mais résolument

\footnotetext{
${ }^{30}$ Discours et messages, p.42, 1.56 à 61

${ }^{31}$ Ibid., loc.cit.

${ }^{32}$ Discours et messages, p.43, 1.59
} 
solidaires. ${ }^{33}$ » L'usage de l'adverbe «très » qui indique l'intensité absolue et sa répétition deux fois avant les adjectifs «libres» et «approfondies» sont non moins révélateurs. Avant de mettre l'affaire dans les mains de la nation "seule juge", de Gaulle termine en assurant qu'en élaborant la nouvelle Constitution, les responsables n'ont "attenté à aucun droit du peuple, ni à aucune liberté publique". Il est à remarquer que l'utilisation du pronom indéfini aucun est assez révélatrice puisque l'auteur aurait pu simplement recourir au pluriel (nous l'avons fait sans attenter aux droits du peuple ni aux libertés publiques). Mais il est incontestable que l'emploi de la forme négative rend le sens beaucoup plus fort que l'emploi de la forme affirmative: d'où sa prédilection de la part de l'auteur.

Si nous examinons ce paragraphe de près, nous trouverons qu'à part les conditions démocratiques dans lesquelles ce travail fut élaboré et dont nous venons de parler, l'atmosphère régnante est celle de la liberté (les Conseils des ministres sont formés d'hommes aussi divers que possibles), de l'égalité (ils n'ont attenté à aucun droit du

\footnotetext{
${ }^{33}$ Ibid., 1.62. Le gouvernement que le général de Gaulle préside comprend, à côté de plusieurs personnalités d'origine non-parlementaire, des membres du parti socialiste (S.F.I.O), du parti radical-socialiste, du M.R.P., du Centre national des Indépendants et Paysans, du Centre des Républicains sociaux. Quatre ministres d'Etat, Guy Mollet (S.F.I.O), Pierre Pflimlin (M.R.P.); Félix Houphouet-Boigny (Rassemblement Démocratique Africain), Louis Jacquinot (Indépendant) manifestent par leur présence aux côté du général de Gaulle le caractère d'union du gouvernement présidé par celui-ci.
} 
peuple) et de la fraternité (les Conseils sont formés d'hommes résolument solidaires). La Constitution est donc faite dans les principes de la Révolution, elle est fidèle à la République.

Cette progression dans la persuasion qui atteint son point culminant avec l'avant dernière phrase (la dernière phrase étant une sorte de péroraison à tout le paragraphe) témoigne de l'art de la rhétorique que possède l'écrivain. En effet, cette progression exige une connaissance profonde et étroite de la manière de réfléchir de l'autre dans ses étapes les plus précises; ceci pour pouvoir suivre ces étapes et combler les lacunes au fur et à mesure en vu de parvenir à une persuasion aussi solide que complète. Autrement dit, l'émetteur doit se mettre à la place du récepteur pour qu'il puisse répondre à toutes les questions que ce dernier peut se poser et dans le même ordre qu'il se les posera: tâche qui, à notre avis, exige beaucoup de talent.

Dans la deuxième partie du discours - où l'incitation à voter "oui" atteint un degré plus élevé dans le ton ascendant - et qui commence à la ligne 96 , le général recourt à une anaphore qu'il utilisera jusqu'à la péroraison: la conjonction "que". Dix phrases qui commencent avec dix "que" sur une page et demi presque. Deux explications à cette anaphore "filée" peuvent être valables: la première relève du domaine du style: l'émetteur veut changer de ton pour fuir une 
monotonie menaçante qui peut résulter de la longueur du texte; la deuxième relève de la pensée même du général.

Cette Constitution, de Gaulle la veut différente des constitutions précédentes. Pour la distinguer de ses semblables, il emploi cette conjonction «Que» qu'il répète au début de chaque phrase. A la constitution nouvelle, une manière nouvelle de procéder. Ainsi, après avoir insisté sur le fait que le projet de Constitution est établi pour le peuple, au lieu de dire «grâce à cette Constitution le pays pourra être dirigé par ceux qu'il mandate» il dit: «Que le pays puisse être dirigé par ceux qu'il mandate. ${ }^{34} \gg$ Il continue, en construisant ses phrases sur le même modèle. Le subjonctif, devenu obligatoire dans la formule choisie donne à la phrase beaucoup plus de force que le présent ou le futur simple qui auraient été utilisés dans les autres formules. Il en va de même pour toute cette série de phrases qui s'étend de la ligne 97 à la ligne140. Optant pour cette formule, le général rend réel la situation dont il parle et donne à ses phrases beaucoup plus de légèreté au niveau du rythme aussi bien qu'au niveau de la structure.

Cependant, les expressions répétées ne sont pas nécessairement des anaphores: elles sont parfois situées au milieu des phrases (comme par exemple l'expression « on la vit » répétée trois fois dans trois phrases successives ${ }^{35}$ ou

\footnotetext{
${ }^{34}$ Discours et messages, p.44, 1.96

${ }^{35}$ Ibid., p.41-42, 1.136-16-20. Cf. Infra., parallélisme syntaxique.
} 
alors en fin de phrase comme par exemple cette épiphore: «C'est donc - dit il - pour le peuple que nous sommes, au siècle et dans le monde où nous sommes, qu'a été établi le projet de Constitution. ${ }^{36}{ }{ }^{2}$ Ici, l'épiphore sert surtout à prouver que, de tous les points de vue, la Constitution paraît convenable. Non seulement elle est destinée au bon peuple mais elle lui est également destinée au bon moment. A part la mise en relief de la proposition principale en vue d'une compréhension plus facile de la phrase surtout pour un discours oral, anaphores et épiphores ont pour but de mieux rythmer la phrase, de la rendre plus harmonieuse et de lui garantir plus de musicalité: effet non négligeable mais, au contraire, recherché par l'auteur.

Néanmoins, quelque soit la place que ces expressions répétées occupent dans la phrase, au début, au milieu ou à la fin, ces répétitions ne perdent rien de leurs effets, d'où l'importance que le général leur accorde ainsi que leur présence intensive dès le début du discours.

Anaphores et épiphores ne sont point le seul genre de répétition dans le discours gaullien. D'autres types de redoublements y sont largement utilisés, et, de par l'importance que leur accorde le général, méritent d'être étudiés. Un de ces types est la redondance.

\footnotetext{
${ }^{36}$ Ibid., p.44, 1.94-95
} 
La redondance dont la définition varie entre "redoublement expressif de l'idée par deux phrases proches $^{37}$ et, "redoublement de l'idée dans deux phrases ou membres de phrase $^{38 "}$ se retrouve sous plus d'un aspect dans ce discours. Sur tous les niveaux, elle ne peut être considérée comme défaut de style. Contrairement à la périssologie qui est un "pléonasme vicieux" et la battologie qui est une "redondance excessive, injustifiée", la redondance rejoint le pléonasme qui recouvre le sens de "surabondance de termes donnant plus de force à l'expression", ou celui de " redoublement de l'idée dans deux mots du même membre de phrase". Tous deux "sont considérés comme des procédés de style; la périssologie et la battologie sont des défauts et ne peuvent avoir d'emploi que (...) dans les textes ironiques ou comiques ${ }^{39 "}$.

Les redondances vont de pair avec les autres éléments de la répétition et ceci dès le début du texte. Examinons quelque exemples:

Se rappelant de l'avènement de la première République de Gaulle dit qu"elle était la souveraineté du peuple, l'appel de la liberté, l'espérance de la justice. ${ }^{40 "}$ En 1848, "on la vit s'élever au-dessus des barricades, se refuser à l'anarchie, se

\footnotetext{
${ }^{37}$ DUPRIEZ Bernard, Gradus, Les procédés littéraires, Edition 10/18, Département d'Univers Poche, Paris, 1984, p.387.

${ }^{38}$ Ibid., p.351.

${ }^{39}$ Ibid., loc.cit.

${ }^{40}$ Discours et messages, p.41, 1.8-9
} 
montrer sociale... ${ }^{41 " ~ D e ~ m e ̂ m e, ~ " l a ~ R e ́ p u b l i q u e ~ s u t ~ r e l e v e r ~ l a ~}$ France, reconstituer les armées, recréer un vaste empire, renouer des alliances solides, faire de bonnes lois sociales, développer l'instruction. ${ }^{42 "}$ Cependant "le régime comportait des vices de fonctionnement (...) qui n'étaient plus compatibles avec les mouvements humains, les changements économiques, les périls extérieurs. ${ }^{43 .}$

Pour inciter le peuple à être "dynamique" et "expéditif" de Gaulle pense qu'il est nécessaire "de rénover l'agriculture et l'industrie, de procurer les moyens de vivre, de travailler, $d e$ s'instruire, de se loger, (...) $d$ 'associer les travailleurs à la marche des entreprises... ${ }^{44}$ " En donnant son approbation à la nouvelle Constitution, le peuple permettra: "Qu'il existe (...) un arbitre national (...) répondant, en cas d'extrême péril, de l'indépendance, de l'honneur, de l'intégrité de la France et $d u$ salut de la République. ${ }^{45, "}$

Cette accumulation de redondances, (note: nous n'avons cité que quelques exemples) montrent à quel point l'auteur des Mémoires d'espoir s'y attache. En fait, les répétitions en général et les redondances en particulier, lui permettent de mieux communiquer avec le récepteur. Celui-ci, attiré en plus par le ton et la diction du général ainsi que par

\footnotetext{
${ }^{41}$ Ibid., p. 42, 1.16-17.

${ }^{42}$ Discours et messages, p.42, 1.21 à 23 .

${ }^{43}$ Discours et messages, p.42, 1.29 à 32 .

${ }^{44}$ Ibid., p.43, 1.77 à 81 .

45 Ibid., p.44, 1.98 à 103.
} 
l'emphase que ce dernier donne à chaque mot prononcé, ne peut se prêter à aucun divertissement.

De nombreux exemples peuvent être relevés dans le discours. Cependant, contrairement aux allitérations et aux assonances, les redondances ne sont point accumulées dans des parties précises: elles se trouvent à tout moment du discours.

Néanmoins, si certains exemples demeurent plus révélateurs que d'autres, ceci est dû, pour la plupart du temps, à la longueur de la phrase. Comme nous venons de le démontrer; plus la phrase est longue, plus elle se prête à un usage plus intense des redondances.

S'apparentant à la redondance, l'énumération aussi est l'une des figures de rhétorique utilisée par le général de Gaulle. A part son effet rythmique, elle a pour rôle de faire un recensement, d'englober les parties d'un tout, de donner plus d'explication en vue d'être plus clair, plus précis ou alors de restreindre sa pensée et la réduire à des limites plus étroites pour éviter la distraction du récepteur.

Ainsi, pour souligner le mérite de la République et obtenir l'adhésion du peuple, de Gaulle «énumère » ses bienfaits:«La République sut relever la France, reconstituer les armées, recréer un vaste empire, renouer des alliances 
solides, faire de bommes lois sociales, développer l'instruction. ${ }^{46}$ »

L'énumération sert aussi à englober les parties d'un tout pour mieux expliquer. Ainsi, Françaises et Français sont appelés à devenir "dynamiques" et "expéditifs" pour pouvoir accomplir la dure tâche qui leur est demandé, à savoir:

"La nécessité de rénover l'agriculture et l'industrie, de procurer les moyens de vivre, de travailler, de s'instruire, de se loger, (...), d'associer les travailleurs à la marche des entreprises. ${ }^{47 "}$

Ne visant souvent que certains domaines spécifiques et en vue d'exclure tous les autres, pour être à la fois bref et précis, de Gaulle recourt à l'énumération pour citer les domaines visés: «...il nous faut, dans les domaines scientifique, économique et social, évoluer rapidement. ${ }^{48}$,

Pour définir le genre de relation qui, d'après la nouvelle Constitution, unira la France aux territoires d'outre-mer et pour insister sur le seul fait que chacun de ces territoires deviendra un Etat et exclure la possibilité que la France les gouverne, de Gaulle englobe toutes les autres disciplines et les cite à la file sans oublier aucune. Ceci, pour préciser les disciplines qui «constitueront un domaine commun»; Ce

\footnotetext{
${ }^{46}$ Ibid., p.42, 1.21 à 23

${ }^{47}$ Discours et messages, p.43, 1.77 à 80 .

${ }^{48}$ Ibid., p.43, 1.70 - 71.
} 
sujet étant un sujet épineux qui ne permet aucun équivoque: «...La politique étrangère, la défense, la monnaie, la politique économique et financière, celle des matières premières, le contrôle de la justice, l'enseignement supérieur, les communications lointaines, constitueront un domaine commun... ${ }^{49}$ »

Pour citer les étapes à suivre pour arriver à des résultats déterminés en ce qui concerne l'Algérie, l'énumération est parfois en gradation; elle est ascendante, progressive : « Le devoir de ramener la paix en Algérie, ensuite celui de la mettre en valeur, enfin celui de régler la question de son statut et de sa place dans notre ensemble, nous imposent des efforts difficiles et prolongés. ${ }^{50}$ »

Les antithèses et les oppositions jouent aussi un rôle non négligeable dans l'exposition des idées. Elles aident l'émetteur à s'exprimer et ceci en rendant les idées plus claires et plus précises. Elles ont essentiellement pour but de cerner un tout, de souligner la différence entre deux énoncés ou plus simplement d'attirer le récepteur pour éviter toute distraction en les utilisant pour alléger le ton. Grâce à ce procédé, l'émetteur est sûr que son idée est saisie, surtout s'il veut rendre une idée plus favorable qu'une autre aux yeux du peuple. Ainsi nous avons des mots tels que « se réformer,

\footnotetext{
${ }^{49}$ Ibid., p.44, 1.126 à 129.

${ }^{50}$ Ibid., p.43, 1.81 à 84.
} 
se briser $^{51} »$, «s'élever/ s'effacer ${ }^{52} »$, « au-dedans/ audehors $^{53} », \ll$ statique/lesmouvements ${ }^{54} »$, , autoritéintérieure/ass uranceextérieure $^{55} »$, , approuvera/repoussera ${ }^{56} »$, «efleurira/p érira ${ }^{57} »$, «accepter/refuser ${ }^{58} »$

Les redondances, l'énumération et les antithèses ne sont mis en relief qu'à la présence d'un rythme. On notera à titre d'exemple, que les mots opposés acquièrent une beauté supplémentaire lorsqu'ils sont régis par un rythme qui les cadence: "approuvera/ repoussera"; "accepter/refuser" et ainsi de suite.

Ce rythme, qui compte beaucoup pour le général et sur lequel le général compte beaucoup a pour fonction de séduire le récepteur, de le rendre - si l'on puisse dire - plus vulnérable en s'adressant à sa sensibilité, à son sens musical. Une fois cette tâche accomplie, la persuasion sera plus aisée, plus profonde.

Le rythme des phrases et la musique qui les imprègne jouent un rôle non négligeable dans la persuasion. Formant une partie inséparable du style, ils accroissent ou atténuent l'influence de l'auteur sur son auditeur. De Gaulle le

\footnotetext{
${ }^{51}$ Discours et messages, p.41, 1.1

52 Ibid., p.42, 1.16-18

${ }^{53}$ Ibid.,17-18

${ }^{54}$ Ibid., p.42, 1.30-31

55 Ibid., p.42, 1.45

${ }^{56}$ Ibid., p.43, 1.65

57 Ibid., 1.91

${ }^{58}$ Ibid.,p.45, 1.134-135
} 
comprend parfaitement. D'où un rythme recherché qui ne peut être le fruit du hasard.

Prenons la deuxième phrase comme exemple ${ }^{59}$. Elle est savamment agencée : deux compléments circonstanciels (ayant chacun presque le même nombre de syllabes) suivis par le sujet (ayant presque le même nombre de syllabes que chacun des compléments circonstanciels) puis deux parties (chacune avec un verbe et un complément d'objet direct ayant chacune le même nombre de syllabes) : donc deux parties non seulement égales, mais aussi ayant la même structure. Pour voir ceci d'une manière plus claire, on pourrait écrire cette deuxième phrase ainsi :

Jusqu'alors

(3 syllabes)

Au long des siècles, (4 syllabes)

L’Ancien Régime

(4 syllabes)

Avait réalisé l'unité

(8 syllabes)

Et maintenu l'intégrité

(8 syllabes)

de la France...

(3 syllabes).

L'exemple des phrases «rythmiques» abonde chez de Gaulle. Conscient de leur effet, il aime et parfois il cherche à en imprégner ses textes. A la ligne 8, c'est le rythme ternaire cher à de Gaulle. Parlant de la République l'auteur dit : «elle était la souveraineté du peuple, l'appel à la liberté,

${ }^{59}$ Ibid.,p.41, 1.3-4 
l'espérance de la justice ${ }^{60} »$. A part l'effet musical que la phrase laisse chez l'auditeur, cette énumération des bienfaits de la République est l'une des techniques de persuasion. La République, qui a pu grâce aux principes qu'elle engendre (souveraineté du peuple, liberté, justice) sauver le peuple autrefois de la «tourmente nationale» et de la «guerre étrangère ", pour reprendre les termes qu'utilise de Gaulle, est toujours la seule qui puisse le conduire au salut.

Ayant toujours pour but d'attirer l'attention de l'auditeur, le rythme ternaire est parfois moins explicite comme dans la phrase suivante: «C'est en un temps où il fallait se réformer ou se briser, que notre peuple, recourut à la République $^{61}{ }$ ou comme dans cette autre: «C'est alors, qu'au milieu de la tourmente nationale et de la guerre étrangère, apparut la République ${ }^{62}{ }^{2}$.

Cette idée de la primauté de la République répétée par l'intermédiaire de deux phrases presque identiques du point de vue structure, dans un intervalle de cinq lignes, ajoute certainement à la beauté du discours. Elle confirme surtout l'idée du général que seule la République peut sauver le peuple.

De Gaulle sacrifie parfois l'ordre logique des mots pour un rythme plus beau, une musique plus légère. C'est alors

\footnotetext{
${ }^{60}$ Discours et messages, p.41, 1.8 .

${ }^{61}$ Ibid., p.41, 1.1 .

${ }^{62}$ Ibid., 1.8 .
} 
que nous pouvons parler de musication. ${ }^{63}$ Il est conscient, lui qui travaille lui-même ses textes avec beaucoup d'assiduité, qu'une fois le texte est crée pour être entendu (même s'il est lu plus tard) les priorités d'exposition sont différentes. Ainsi, à la ligne 13, parlant de la première République, dans la phrase «on la vit révolutionnaire et guerrière, renverser trônes et privilèges» l'ordre logique est renversé au profit du son «è» que l'on trouve dans « révolutionnaire», «guerrière " et « privilèges ». L'ordre logique aura été « révolutionnaire et guerrière, renverser privilèges et trônes ». De cette manière, le premier mot de la première partie de la phrase conviendrait au premier mot de la seconde et le second au second.

Autre remarque concernant cette même phrase: « on la vit révolutionnaire et guerrière, renverser trônes et privilèges ». Favorisant le rythme au détriment du sens, de Gaulle utilise le verbe "renverser" pour les deux mots "trônes" et "privilèges". Or, le verbe "abolir" serait plus approprié au mot "privilèges" pour désigner la suppression de ces derniers. De Gaulle le sait, mais pour des raisons poétiques, il insiste à garder la phrase telle quelle. De par son rythme et sa métrique parfaitement étudiés, la phrase s'approche effectivement des vers poétiques. Les deux

\footnotetext{
${ }^{63}$ (Néol.) donner à l'aspect sonore du texte priorité sur les autres aspects,
} notamment sur le sens. Gradus, op.cit. p.304. 
membres de la phrase ainsi dessinés, seront parfaitement égaux du point de vue nombre de syllabes :

ré- vo- lu- tio- nair et- guer- yère $(8$ syllabes)

ren- ver- ser- tro- né- pri- vi- lège $(8$ syllabes).

Toutefois, dans le discours gaullien la musique s'obtient non seulement du rythme mais également des mots. Le général agence les mots les uns près des autres selon leur sens, leur rythme mais aussi selon les lettres qui les forment. C'est ainsi que nous obtenons des "retours multipliés de sons

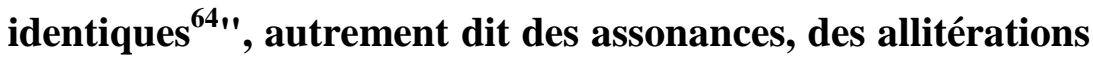
et des homéotéleutes. Comme le propose Morier, nous allons réserver allitération aux répétitions «de consonnes, notamment des consonnes initiales, mieux perçues et souvent mises en évidence par l'accent affectif » et assonance aux "répétitions des voyelles. ${ }^{65 "}$ Quant à l'homéotéleute ce sera la rime ou l'assonance introduite dans la prose puisqu' «on place à la fin des phrases ou des membres de phrases des mots de la même finale. ${ }^{66}$ »

Les consonnes les plus répétées sont les suivantes: "p", "b", "s", "m" et "f". Les voyelles au son "é" -quelque soit sa typographie - est la plus répétée.

\footnotetext{
${ }^{64}$ Gradus, op.cit. p.33.

65 Ibid.,p.34.

${ }^{66}$ Ibid., p.232.
} 
Les allitérations et les assonances s'accumulent dans leur majorité dans les premier et avant dernier paragraphes du discours juste au moment où le texte change de nature: de texte purement argumentatif, il se transforme en texte injonctif. Ceci est dû au rôle qui leur est assigné par le général : ajouter une note musicale, produire un effet rythmique aux deux moments décisifs du discours: au début où le plus important c'est d'attirer l'attention du récepteur pour qu'il arrive le mieux à suivre l'émetteur et à la fin du discours pour laisser la meilleure impression chez le récepteur.

Examinons le premier paragraphe ou allitération et assonance abondent ${ }^{67}$ :

(nous mettrons al pour désigner l'allitération, as pour assonance et hom pour homéotéleute)

1.1...........................se former ou se briser (hom)

1.2................notre peuple pour la première fois (al)

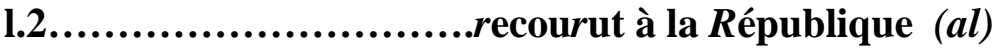

1.3........................Jusqu'alors au long des siècles (al)

1.4..............réalisé l'unité et maintenu l'intégrité (hom)

\footnotetext{
${ }^{67}$ Nous avons pensé au début regrouper l'allitération par consonne et l'assonance par voyelle et les homéotéleutes à part ce qui aurait été plus ordonné voire plus clair mais nous avons renoncé à ce faire, le but de ce recensement étant de souligner le nombre total de ces procédés et de montrer leur accumulation, afin de souligner l'importance du rôle que le général leur accorde dans le discours.
} 
1.5........une vague de fond se formait dans les profondeurs (al)

l.6.............hors d'état de s'adapter à un monde (as \& al)

(Remarquons l'alternance des sons "d", "é", "a" d'où la ressemblance entre les mots "d'état" et "s'adapter").

I.8..................au milieu de la tourmente nationale (al)

1.8 . .apparut la République(as \& al)

(Remarquons l'alternance des sons "p", "r", "u" d'où la ressemblance entre les mots "apparut" et "République").

1.8-9.............................. souveraineté du peuple, l'appel de la liberté, l'espérance de la justice (as \& al)

(Remarquons l'alternance des sons "é", "p" et "l", aussi bien que la ressemblance entre les mots "peuple" et "appel" surtout que les deux mots se suivent).

1.12-13...... des formes diverses, au cours de ses règnes successifs (as \& as)

(Remarquons l'alternance des sons "r" et "s")

l.15............................les abus et les troubles (al)

1.16-17......se lever (......), se refuser (.....), se montrer (...) (hom)

(Remarquons aussi que les trois verbes utilisés l'un après l'autre sont des verbes pronominaux). 

.sociale au-dedans et fraternelle au dehors » (hom)

1.21 à 1.24.. la République sut relever la France, reconstituer les armées, recréer un vaste empire, renouer des alliances solides, (...) développer l'instruction. Si bien qu'elle eut la gloire d'assurer.............. (as, al \& hom)

(Remarquons l'alternance des sons "r" et "é". Autre remarque: pour la plupart des mots le son "r" est au début du mot, il précède le son "é" qui, lui, vient à la fin du mot.

Il en est de même pour l'avant dernier paragraphe où les allitérations et les assonances abondent aussi. Ceci à la seule différence près, que les consonnes répétées ne sont pas les mêmes qu'au premier paragraphe. Autrement dit, au lieu que les consonnes les plus répétées soient les consonnes " $r$ " et "s", celles que nous trouvons surtout dans l'avant dernier paragraphe sont les consonnes "m" et "p"(bien que les "s" soient également présents). En voici le recensement:

L. 136-139....Devenu membre de la Communauté, il pourra (...) après s'être mis d'accord, avec les organes communs, assumer son propre destin, indépendamment des autres................................... (al) 
L.143-145...De cette façon, pourront être prises, sur mandat donné par le peuple, les dispositions nécessaires à la mise en place des nouvelles institutions............................................. (al)

L.145-146....Voilà (...) de quoi s 'inspire et en quoi consiste la Constitution qui sera, le 28 septembre, soumise à vos suffrages..................... (al)

Le général a également recours à un procédé assez rare de la rhétorique à savoir l'isolexisme. Celle-ci est l'une des dérivés de la paronomase, qui est le rapprochement de mots dont le son est à peu près semblable mais dont le sens est différent. Si les vocables appartiennent au même lexème, c'est une isolexisme. $^{68}$

Ainsi, parmi les qualités de la Constitution à voter, c'est qu'elle permettra de mettre en place "un gouvernement qui soit fait pour gouverner". L.104 Cette phrase, bien qu'elle soit d'une simplicité parfaite, d'une sobriété étonnante est remarquablement riche de sens et de connotation. Se rapprochant, de par ses deux qualités paradoxales, des proverbes, nous dirions même des maximes, elle témoigne d'une maîtrise profonde de l'art de la rhétorique.

${ }^{68}$ Gradus, op.cit.,p.332. 
Cependant, l'art de la rhétorique implique la présence d'autres éléments qui, s'ils figurent dans le texte et sont bien utilisés, une complète adhésion de la part du récepteur sera garantie. Parmi ces éléments: le choix des mots. En effet, de Gaulle choisit ses mots avec une grande finesse. Même si ces mots paraissent parfois exagérés, c'est cette exagération qui leur permet, paradoxalement, de rejoindre les autres procédés de la rhétorique pour atteindre le but visé. N'oublions pas que tout texte politique doit s'apparenter à la grandiloquence, c'est un texte emphatique de par sa nature surtout s'il est oratoire; toute modestie "personnelle" de la part de l'homme politique -du moment qu'il parle au nom de son pays - n'est pas nécessairement bienfaisante. Quant à la modestie "'nationale", elle lui est complètement défendue surtout si c'est un texte oratoire. Le discours politique doit nécessairement s'imprégner d'ordre, de mesure, de clarté donc de classicisme. Entre ce qu'il faut dire, la grandiloquence dans ce qui est dit et la personnalité même de l'homme d'Etat, réside tout l'art de la rhétorique ${ }^{69}$.

Le mot pour de Gaulle compte beaucoup. Ceci apparaît, même pour un lecteur ou un récepteur non avisé, dès les premières lignes. De Gaulle dit: "C'est en un temps ou il fallait se réformer ou se briser, que notre peuple (...)

\footnotetext{
${ }^{69}$ Il suffit de prendre l'exemple de Dominique de Villepin et de Colin Powell le 14 février 2003au Conseil de Sécurité lors du vote pour ou contre la guerre de l'Iraq, pour voir la différence entre un diplomate grandiloquent classique et un homme d'Etat tout à fait ordinaire.
} 
recourut à la République. ${ }^{70 "}$ Le choix des verbes de la première phrase est assez révélateur: «se réformer» et «se briser» sont utilisés en tant que contraires. Est-ce vraiment des contraires? Si le peuple échoue à se réformer, le second choix serait-il de «se briser»? La réponse étant non, l’idée est claire: Françaises et Français doivent voter «oui» à la Constitution donc accepter l'avènement de la Vème République. Sinon, la France sera non pas "arriérée" mais "brisée". Tout sera ruiné, c'est le renouveau ou le néant. C'est le « tout ou rien » d'Antigone.

Le choix des mots utilisés avec "la république" est d'une extrême finesse. Il suffit de lire les vingt premières lignes pour en être convaincu. Dans cette partie, de Gaulle brosse l'histoire des quatre républiques françaises. Dans ce panorama qu'il dresse, il laisse -malgré lui - libre cours à ses sentiments favorables envers la république. Néanmoins, derrière sa description des événements transparaît une subjectivité que trahissent les mots choisis. Selon lui, toutes ces républiques françaises avaient de bonnes intentions et devaient, par les principes humains qu'elles engendraient, conduire le peuple vers le salut. Des mots comme «révolutionnaire ${ }^{71} »$, des verbes comme «s'élever ${ }^{72} »$, «s'offrir ${ }^{73} »$ marquent l'élévation de la République, le rang

\footnotetext{
${ }^{70}$ Discours et messages, p.41, l.1-2.

${ }^{71}$ Ibid., p.41, 1.13

${ }^{72}$ Ibid., p.42, 1.16

${ }^{73}$ Ibid., p.42, 1.20
} 
bien distinct qu'elle occupe aux yeux du général de Gaulle et l'action bienfaisante de celle-ci. Par le choix de ces mots, de Gaulle insiste sur le rôle de la République comme sauveur, surtout après des mots et expressions comme «profondeurs » qui évoque l'obscurité, la peur ${ }^{74}$ et «tourmente nationale $^{75}$ » qui évoque le trouble et l'instabilité.

De Gaulle se montre partisan farouche de la République et veut absolument que le peuple le soit également, il croit à la puissance $^{76}$ délimitée de la République, insiste à souligner son rôle de sauveur, d'où le recours à une expression exagérée, une hyperbole «réparer le désastre. ${ }^{77}$ " L'exagération est claire: seule une faute, une erreur peut être réparée mais un désastre, plus grave et beaucoup plus sérieux serait plutôt « comblé ».

Nous remarquons que les bâtisseurs de ces Républiques sont totalement négligés, ce sont les Républiques qui sont de par elles-mêmes révolutionnaires. Ce sont elles-mêmes qui se fixent les buts et qui décident de leur sort. L'accumulation des verbes pronominaux «s'élever ${ }^{78}$, «se refuser $^{79} »$, «se montrer ${ }^{80} »$, «'offrir ${ }^{81} »$ n'en sont qu'une preuve évidente.

\footnotetext{
${ }^{74}$ Ibid., p.41, 1.5

75 Ibid., p.41, 1.7

${ }^{76}$ Nous insistons sur ce mot de puissance et non de pouvoir car nous tenons à ce sens de force et d'énergie par opposition a celui d'autorité.

${ }^{77}$ Discours et messages, p.42, 1.20 .

${ }^{78}$ Ibid., 1.16

${ }^{79}$ Ibid., 1.17

${ }^{80}$ Ibid.,loc.cit.

${ }^{81}$ Ibid., 1.20
} 
Même lorsque le général mentionne le déclin, la ruine même de ces Républiques, il n'hésite pas à recourir de nouveau à un verbe pronominal, celui de « $s^{\prime}$ effacer. ${ }^{82}{ }$ ”

Remarquons la beauté, la légèreté du verbe «s'effacer », du point de vue sens par rapport au verbe «s'effondrer » que de Gaulle n'a pas utilisé mais qui aurait été plus approprié dans ce cas. Cependant, nous devons souligner que, dans ce discours, chose curieuse, la seule fois où le général utilise le mot «effondrement ${ }^{83}{ }$ " il l'associe à la IVème République. Cette fois-ci, de Gaulle utilise un mot fort de connotation. Il veut être grave, parce que la priorité maintenant, ce n'est plus à l'utilisation d'un mot qui garde à la République sa dignité, son honneur, (rappelons-nous du verbe $s^{\prime}$ effacer, le verbe «succomber ${ }^{84}{ }$, l'expression «ne peuvent pas maîtriser ») mais plutôt à inciter le peuple à voter «oui» à la Vème République.

Cette image des Républiques «hautaines » qui n'ont besoin de personne et dont tout le monde a besoin, qui se veulent maîtresses de leur sort et qui ne désistent point, évoquerait «Le sacre de Napoléon » célèbre tableau de David où, dans un acte solennel et très significatif, Napoléon prit la couronne qui lui était destinée d'entre les mains du Pape et se la mit lui-même sur la tête.

\footnotetext{
82 Ibid., 1.18

83 Ibid., 1.48

${ }^{84}$ Ibid., 1.14
} 
De Gaulle est épris de la République. Il veut surtout que ses auditeurs le soient. Il est décidé à ne laisser donc s'échapper aucune occasion sans nous faire remarquer, une fois de plus, le beau visage, les belles qualités de la République. Ainsi, la cause du déclin des différentes Républiques françaises n'est jamais mentionnée. Ces Républiques ne sont jamais effondrées parce qu'elles se sont écartées des principes qu'elles avaient déjà prêchés et qui étaient la raison de leur avènement (Bien que ceci soit vrai, au moins pour la première République). Si les Républiques échouent à conduire le peuple vers le salut c'est parce que les circonstances étaient devenues défavorables à la continuation de ces Républiques: d'où l'utilisation des verbes tels que «succombent ${ }^{85}$ » ou alors « $s^{\text {'effacent }}{ }^{86}{ }^{8}$ ou enfin une expression telle que « ne peuvent pas maîtriser. ${ }^{87}$ »

Dans le deuxième paragraphe, de Gaulle choisit un mot qui lui a valu certains reproches. En énumérant les bienfaits de la IV ème République, le général évoque la reconstitution "des armées". Jean-François Revel commente cet emploi en disant:" en français moderne, on dit l'armée et les diverses $\operatorname{armes}^{88 " .}$. Or, l'emploi du pluriel pour le singulier est un genre de synecdoque. C'est le pluriel emphatique. De même, cet emploi du pluriel pour le mot "armée" et précisément

\footnotetext{
${ }^{85}$ Discours et messages, p.42, 1.15

${ }^{86}$ Ibid., p.42, 1.18

${ }^{87}$ Ibid., p.42, 1.16

${ }^{88}$ Revel Jean-François, op.cit. p.85.
} 
par le général est justifié. On connaît l'histoire de ce chef charismatique. On sait que le général de Gaulle a fait le métier des armes, qu'il était pendant longtemps chef de la France libre. On sait combien il fut fier de sa carrière militaire et comment il admirait l'armée et le métier des armes. Il suffit de rappeler que le mot "armée" figure dans le titre de deux de ses ouvrages les plus connus: Vers l'armée de métier et La France et son armée. Si l'on ajoute à cela l'atmosphère même du discours, et le désir d'impressionner les récepteurs, l'emploi du pluriel serait tout à fait accepté.

Ceci n'empêche point que le général préfère le pluriel et qu'il aime l'employer chaque fois que c'est possible. Cet emploi peut parfois paraître abusif. De nombreux exemples dans le discours en témoignent. Il en a inlassablement recours surtout lorsqu'il évoque les différents défis que l'Etat doit affronter tels que «les événements terribles de $1940^{89} »$, «les forces gigantesques (qui) sont en train de transformer le monde..$^{90} \gg$ Ceci pour montrer l'importance primordiale d'une République puissante, qui soit capable d'affronter ces grands défis. En cas de vote pour cette République, et pour reprendre les mots du général, celle-ci comportera des vertus de fonctionnement qui seront cette fois-ci compatibles avec «les mouvements humains, les

\footnotetext{
${ }^{89}$ Ibid., p.42, 1.34 .

${ }^{90}$ Ibid., p.43, 1.69
} 
changements économiques et les périls extérieurs ${ }^{91}$. " L'usage du pluriel est donc justifié : rien de plus important que de voter pour un régime puissant qui soit à la hauteur des difficultés affrontés.

Dans le quatrième paragraphe, après avoir dressé l'histoire des Républiques précédentes, le général termine le paragraphe par l'emploi d'un mot assez fort de connotation. C'est le mot de «paralysie». Si le résultat du référendum est négatif, l'Etat ne risque ni de «succomber» ni de «s'effacer» mais de vivre handicapé. Ainsi, deux explications se prêtent pour justifier ce choix: la première explication est que de Gaulle, pour des raisons sentimentales, n'arrive pas à lier la République qu'il chérit à un verbe tel que celui de "ruiner", "s'effondrer" surtout que ces mots qui marquent une fin déterminée ne sont guère employés tout au long du texte. La seule fois où de Gaulle parle d'effondrement ce n'est qu'une menace. ${ }^{92}$

La seconde explication est la suivante: le général met en garde les Français contre le refus de la Constitution. Il leur défend catégoriquement de voter "non", si oui, le pays sera condamné à une vie d'handicapé, donc une vie pire que la mort, car la mort, elle, est bienfaisante. Elle renferme en quelque sorte le germe de la vie, et le phonix une fois mort

\footnotetext{
${ }^{91}$ Discours et messages, p.42, 1.31-32.

${ }^{92}$ Ibid., p.42, 1.48.
} 
renaît de son cendre, plus grand et plus fort. Ce ne sera pas le cas de la République.

Quant au choix du mot «univers ${ }^{93}{ }$ il a lui aussi valu à l'auteur une critique amère. Jean-François Revel pense que ce mot « ne s'emploie pas aujourd'hui qu'en astronomie et dans le langage d'une certaine critique d'art ou littéraire » et que «le monde, la planète; l'humanité seraient encore excessif, quoique d'un usage normal». Il pense que de Gaulle "imite quelqu'un ou quelque chose que d'aller chercher un tel mot". Revel songe à Auguste:

Je suis maître de moi comme de l'univers.

Selon lui, de Gaulle archaïse, il imite la langue du XVIIème siècle et de commenter: "C'est plus qu'une affectation du style et de la langue, affectation qui, à elle seule exigerait qu'on cherchât les sources dans la psychologie du personnage. ${ }^{94 "}$

Nous avons déjà admis que de Gaulle fait un usage parfois particulier de certains mots emploie d'autres qui semblent "archaïques". Mais le fait est là: de Gaulle appartient a la bourgeoisie cultivée; on ne connaît chez les de Gaulle que des procureurs et des hommes de loi qui passent pour de fins lettrés: le grand-père paternel a écrit Une vie de Saint-Louis, son épouse publia des romans

\footnotetext{
93 Ibid., p.43, 1.87.

${ }^{94}$ REVEL, Jean-François, op.cit., p.26-27.
} 
moraux (Adhémar de Brescastel), des essais, des biographies, et dirigea la Correspondance des familles, le père est à la fois docteur ès lettres, ès sciences et en droit, et à 84 ans, fait encore pour s'amuser des problèmes de géométrie.

De Gaulle, lui, est un homme de lettres. Contrairement à la majorité des hommes politiques, il écrit lui-même ses discours, ses messages et même ses livres. Aussi, jouit-il d'un grand pouvoir de conviction et d'une grande maîtrise de l'art de la rhétorique. Travailleur acharné qui refuse tout "nègre", de Gaulle travaille laborieusement ses textes. Il les rature tous abondamment. Il rédige avec un soin extrême des ouvrages qu'il veut fondamentaux et qui le sont presque tous: La Discorde chez l'ennemi, Le Fil de l'épée, Vers l'armée de métier, La France et son armée et après la guerre, ses Mémoires de guerre en trois volumes puis ses Mémoires d'espoir. Ses lettres, discours et messages recueillis par son fils, occuperont plus de vingt volumes. Bien sûr « il est d'une ponctualité qui confine à la manie. ${ }^{95}$ "

Imprégné de littérature classique, il a lu tous les auteurs classiques et a une prédilection pour Bossuet. Il a subi l'influence des grands classiques, et s'est inspiré sûrement de leur manière d'écrire voire même de penser. Mais jamais il

\footnotetext{
${ }^{95}$ CONTE, Arthur, Bâtisseurs de la France de l'an 1000 à l'an 2000, Plon,
} Paris, 2004, p.495.. 
ne les copia. Et, malgré l'abondance de son œuvre écrite, la moindre phrase est de lui.

Ce discours du général n'est point dénudé des mots signés de Gaulle, ou tout au moins des mots chers à de Gaulle. Ces mots sont révélateurs et encourageants. Accumulés pour la plupart dans la partie ou le chef charismatique expose son projet, ces mots font la synthèse du discours et contribuent à réaliser l'objectif du discours : celui d'orienter un vote: pour ou contre une nouvelle constitution.

Ils sont révélateurs de la pensée de l'émetteur puisqu'ils expliquent aux récepteurs ce que sera l'image de la France une fois le projet de la Constitution accepté. Ils sont encourageants vis-à-vis du peuple dans la mesure où ils lui permettront d'opter pour la nouvelle Constitution en étant largement convaincu et en se sentant plus à l'aise.

Dans cette partie ou le chef charismatique expose l'essentiel de son projet, de Gaulle a recours à des mots à la fois révélateurs et encourageants. Révélateurs en ce qui concerne l'explication de cette nouvelle Constitution et encourageants vis-à-vis du peuple qui devra se sentir plus à l'aise en optant pour le «oui». Grâce à cette Constitution, la France jouira de «l'indépendance», «l'honneur», «l'intégrité. ${ }^{96} »$ Le pouvoir revêtira une «structure

\footnotetext{
${ }^{96}$ Discours et message, p.44, 1.102
} 
équilibrée. ${ }^{97}{ } \ll \mathrm{La} \quad$ compétence $», \quad$ «la dignité $»$, «l'impartialité ${ }^{98} »$ de l'Etat seront mieux garanties.

Les mots chers et propres à de Gaulle sont baptisés ainsi parce qu'ils sont inlassablement répétés dans les écrits gaulliens et propres parce qu'ils revêtent dans l'esprit du général un sens assez particulier, assez précis, un sens qui lui est propre. Ces mots sont «assumer ${ }^{99}$ » qui est «l'un des plus fréquents parmi les verbes gaulliens ${ }^{100}$ » et qui, en fait, incarne parfaitement le caractère du général. Nous devons aussi souligner les mots de «vocation $^{101}{ }^{\text {», « }}$ salut ${ }^{102}$ » et " grandeur $^{103}$ » qui eux étaient, pendant les années soixante, signés de Gaulle. Le peuple français qui avaient tant souffert pendant la Seconde Guerre mondiale et jusqu'à l'avènement de la Vème République avait besoin d'un vocabulaire différent. Voulant rejeter à jamais le vocabulaire de la faiblesse, de la défaite et de l'humiliation, il se mit d'accord avec son chef pour tourner la page et aspirer dorénavant à une nouvelle vie qui serait symbolisée par l'utilisation d'un nouveau vocabulaire, un vocabulaire qui sera imprégné d'espoir et d'élan vers le bonheur Un vocabulaire qui soit le symbole d'une nouvelle époque où la «vocation» de la

\footnotetext{
${ }^{97}$ Ibid., 1.112

98 Ibid., 1.122

${ }^{99}$ Ibid., p.45, 1.138

${ }^{100}$ REVEL, Jean-François, op.cit.,p.38.

${ }^{101}$ Ibid., p.43, 1.90

${ }^{102}$ Ibid., p.42, 1.25

103 Ibid., p.45, 1.155
} 
France la conduira au «salut» autrement dit à la « grandeur ».

Les parties solennelles du discours sont renforcées par des métaphores telles que celle de la troisième phrase. ${ }^{104}$ De Gaulle dit:

«Mais tandis qu'une immense vague de fond se formait dans les profondeurs, (le pays) se montrait hors d'état de s'adapter à un monde nouveau. C'est alors qu'au milieu de la tourmente nationale et de la guerre étrangère apparut la République ». L'auteur a recours à une métaphore filée qui met au point tout un champ lexical de la mer : la « vague » de «fond» qui se forme dans les «profondeurs». Cette métaphore atteint son point culminant avec le mot « tourmente » très bien choisi car il revêt deux sens différents, qui, tous deux, conviennent parfaitement à la phrase. Le premier sens «tempête violente» vient couronner la métaphore filée où la mer est omniprésente et le deuxième sens qui est celui de « troubles graves » convient non à l'image mais au côté réel de la phrase. La phrase peut donc être lue ainsi :

- C'est alors qu'au milieu de la tempête violente apparut la République ;

- C'est alors qu'au milieu des troubles graves apparut la République.

${ }^{104}$ Ibid., p.41, 1.4 à 8 
Grâce à sa connaissance approfondie du vocabulaire français, de Gaulle arrive à choisir un mot qui convient à la fois à la métaphore qu'il s'est choisie et à la phrase formulée en dehors de la présence de l'image métaphorique.

De Gaulle a recours à une autre métaphore pour montrer que le projet de la Constitution a été fait «sans avoir attenté à aucun droit du peuple». Ce verbe, à notre avis est formidablement choisi. Le général considère la moindre violation d'un droit du peuple comme étant non pas un crime mais un attentat. Cette métaphore où le droit du peuple est personnifié élève la Constitution au rang des lois sacrées où les moindres crimes ne sont ni tolérés ni pardonnés.

L'autorité judiciaire est personnifiée du moment qu'elle est "la gardienne de la liberté. 105 "

Avant de terminer, de Gaulle interpelle les Françaises et les Français, dans une dernière tentative d'emporter leur adhésion et leur demande de tout son cour au nom de la France de répondre: OUI! ${ }^{106}$. Il appelle une dernière fois en eux le nationalisme et l'amour de la patrie. Cette patrie qui leur demande de la sauver. L'art de la rhétorique atteint son paroxysme et de Gaulle, après cette longue plaidoirie peut être sûr du résultat du vote. Nous le sommes au moins! Il termine son discours par une note claire, une métaphore où

\footnotetext{
${ }^{105}$ Discours et messages, p.44, 1.121

${ }^{106}$ Nous paraphrasons de Gaulle.
} 
l'espoir est assimilé au soleil: "un grand espoir se lèvera sur la France"; soleil qui se lève après une longue nuit ou, comme le veut de Gaulle après une "tourmente. ${ }^{107 " . ~ D e ~}$ Gaulle ouvre et clot le discours par deux métaphores empruntée à la nature: la première renferme l'angoisse et la peur, la dernière l'espoir et le bonheur.

Les figures se mêlent en bouquet final: aux métaphores s'ajoutent les répétitions, les énumérations, les antithèses, la personnification. Toutes ces figures s'enchevêtrent, elles renforcent l'argumentation et en même temps elles font appel à l'émotion et à la fierté nationale dans l'envolée finale. On peut, comme nous l'avons déjà mentionné, trouver ce style grandiloquent, mais il faut tenir compte de l'occasion dans laquelle ce discours est prononcé et il est authentique car il correspond à une croyance profonde du chef charismatique.

Le résultat de ce discours prouve que la rhétorique du chef charismatique a porté ses fruits: nous savons que les Français ont voté "oui" à la constitution proposée et que celle-ci est toujours en vigueur. Nous savons également que Charles de Gaulle fut le premier président de la $V^{\text {ème }}$ République et que celle-ci continue à demeurer.

Sans tenir compte du résultat du discours, on ne pourra nier l'effort que ce chef charismatique a déployé pour

${ }^{107}$ Allusion à la métaphore qui ouvre le discours. 
mettre à jour un texte aussi travaillé. A la manière des grands latins, de Gaulle attacha la plus grande importance au plus petit détail. Ceci transparaît derrière chaque phrase, chaque mot et chaque lettre. En fait, de Gaulle a pu donner un discours qui rappelle les grands auteurs classiques. Il suffit de lire le témoignage de Françoise Boursin, professeur à l'université Paris-Sorbonne faisant l'éloge de l'ex-premier ministre français Dominique de Villepin, "disciple du

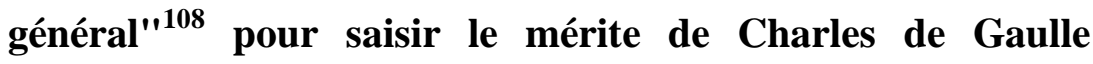
l'écrivain.

Ceci dit, il nous paraît parfaitement appliqué à l'auteur du Fil de l'épée un commentaire qui vient du Monde (16 au 17 février 2005) dans l'article intitulé" Tantôt encensé, tantôt dénigré: Dominique de Villepin ou le refus de la fatalité":

"Personnalité peu commune dans le monde diplomatique, il irrite certains autant qu'il fascine les autres, quand ce ne sont pas les mêmes. Son côté "quand on veut on peut", "il n'y a pas de fatalité", "des hommes, des idées peuvent faire bouger les choses", déplacer les montagnes, résoudre les conflits, séduit ou déconcerte ceux qui sont habitués à des exposés de doctrine plus classiques. De même que ses discours, il aime à mêler les poètes. ${ }^{109 ", ~}$

\footnotetext{
${ }^{108}$ BOURSIN Françoise, art.cit. p.106.

109 "Tantôt encensé tantôt dénigré: Dominique de Villepin ou le refus de la réalité", in Le Monde, 16 au 17 février 2005.
} 


\section{Bibliographi}

BOURSIN Françoise, Dominique de Villepin et Colin Powell: deux rhétoriques face à face, in Communication et langages, 2005, $\mathrm{n}^{\circ} 145$, pp.95-106.

CONTE, Arthur, Bâtisseurs de la France de l'an 1000 à l'an 2000, Plon, Paris, 2004.

DE GAULLE, Charles, Discours et messages, T.III, Avec le renouveau, 1958- 1962, Plon, Paris, 1970.

DUPRIEZ Bernard, Gradus, Les procédés littéraires, Edition 10/18, Département d'Univers Poche, Paris, 1984.

C. PEYROUTET, Style et rhétorique, Nathan, Paris, 1998.

REVEL, Jean-François, Le style du général, Edition René Julliard, Paris, 1959.

ROMILLY, Jacqueline de, Les grands Sophistes dans l'Athènes de Périclès, "Biblio Essais", Editions de Fallois, Paris, 1988.

"Tantôt encensé tantôt dénigré: Dominique de Villepin ou le refus de la réalité, in Le Monde, 16 au 17 février 2005 és de rhétorique basé sur la répétition de sons identiques exige beaucoup de talent. Même si de Gaulle était loin de connaître le nom de chacun de ces procédés, il lui suffit d'avoir savamment su les utiliser. En avoir fait un usage médiocre, aurait tourné toute cette atmosphère de grandiloquence en un comique sarcastique. 
Nouveau (1.5), renouveau (1.19), nouvelle (1.37, 54, 144), rénover (1.77), rénovera (1.132).

RACINE, Andromaque, V, 5, v.1638 cité par Gradus).

En effet, la musique qui émane des textes oratoires du général confère à ces textes non seulement une légèreté, une beauté gaulliens est un facteur essentiel indissociable non seulement de la compréhension vraiment décontournable. cadence es allitérations abondent dans les textes du général surtout dans les textes oratoires. écrits du général surtout quand celui-ci chez de Gaulle

\section{Les Métaphores}

Les métaphores aussi sont présentes dans les écrits du général de Gaulle. Les images empruntées à la mer lui sont particulièrement chères. On les trouve partout (que ce soit dans ses lettres, ses discours ou ses messages). La mer, avec tout ce qu'elle évoque de calme, de douceur de peur et d

Grand nombre d'éléments contribue à rythmer le texte gaullien en général et ce discours en particulier. L'un de ces éléments est le rythme ternaire, cher à de Gaulle.

\section{Conclusion du Rythme}

Nous sommes en plein domaine de la musique, voire même de la métrique et de l'harmonie poétique. 


\section{Enumération}

De même, grâce à la nouvelle constitution, "la compétence, la dignité, l'impartialité de l'Etat en seront mieux garanties." L.121-122

Au sein de la Communauté,

"chaque territoire va devenir un Etat qui se gouvernera lui-même, tandis que la politique étrangère, la défense, la monnaie, la politique économique et financière, celles des matières premières, le contrôle de la justice, l'enseignement supérieur, les communications lointaines constitueront un domaine commun...".L.124 à 129.

\section{Conclusion Rythme}

Considérées comme des écarts usuels de la répétition, les anaphores et les épiphores ne sont pas les seules à figurer dans ce discours du général. D'autres types de répétition qui relèvent cette fois-ci du domaine de la musique, nous dirions même de la métrique - (bien que le mot métrique soit réservé au domaine de la poésie du moment qu'il est attribué à tout système de versification et bien que la métrique tout court soit la science qui étudie les vers, nous tenons à employer ce mot en étudiant la prose du général, tellement celle-ci est imprégné de rythme, de cadence et de musique), y sont également présents. Quoique ces derniers n'aient pas de rôle, de par leur nature, au niveau de la compréhension du 
texte, ils ne sont guère moins importants. Dans un texte oratoire, faire résonner le texte, par des "retours multipliés de sons identiques" (gradus p. 33), est déjà l'un des atouts les plus importants pour attirer l'attention du récepteur.

Il faut cependant préciser qu'allitérations et assonances qui sont des «retours multipliés d'un son identique» (Gradus, les procédés littéraires (Dictionnaire) Bernard Dupriez, p.33) sont largement utilisées par le général de Gaulle. Dans ce discours, bien que leur rôle soit réduit à faire résonner le texte, ce rôle est suffisamment important vue la qualité oratoire du texte. (nous insistons sur ce point car souvent l'allitération est la reproduction allusive d'un son comme dans le célèbre exemple des serpents de Racine : «Pour qui sont ces serpents qui sifflent sur vos têtes ?.... 


\section{AUTRES}

\section{Figures expressives}

PARONYMES mots presque homonymes.

CADENCE Harmonie qui résulte de l'arrangement des mots;

ECHO SONORE je suis allé au marché des oiseaux.

\section{ECHO SONORE et HARMONIE}

Différence entre écho sonore et harmonie : dans l'écho sonore les rapprochements sont crées par des sons identiques ; dans l'harmonie, tous les sons vocaliques entrent en jeu et peuvent combiner les harmoniques de leurs formants.

Grammont conçoit surtout la mise en correspondance par un son commun:

Le groupe est «progressif» si le son commun est le dernier:

(Et ce fut là-dessus);

«régressif » s’il est le premier:

(La comtesse à son bras s'appuyait...)

«embrassé» s'il est au milieu:

(C'est que l'un est la griffe et que l'autre est la serre.) 
ANTITHESE Présenter mais en l'écartant ou en la niant, une idée inverse, en vue de mettre en relief l'idée principale.

Ex.: D'autres préfèrent le monologue intérieur, moi non, j’aime mieux battre.

Autre ex.: Le Canada est le paradis de l'homme d'affaires, c'est l'enfer de l'homme de lettres.

:exagération dans le ton, dans les termes employés, dans les manière.

SYNECDOQUE Trope qui permet de désigner qqch par un terme dont le sens inclut celui du terme propre ou est inclus par lui. (La synecdoque introduit une distance ce qui permet divers effets).

Ex. de littré : une voile pour un navire.

Rem. 1 Fontanier énumère de nombreuses variétés de synecdoque:

1 La partie pour le tout : une voile pour un navire.

2 La matière pour l'objet: Vous êtes le sang d'Astrée (pour son fils).

3 Le nombre.

Le singulier pour le pluriel : L'ennemi (pour les ennemis).

Le pluriel pour le singulier: il fut loin d'imiter la grandeur des Colbert. C'est le pluriel emphatique ; (v. grandiloquence). 
ANTONOMASE Prendre un nom commun pour un nom propre, ou un nom propre pour un nom commun. LITTRE. L'antonomase agrémente le style. Elle correspond, comme dit Barthes, à qqch de mythique.

\section{Ex.: Un Harpagon (pour un avare)}

Il y a des antonomases très spontanées :

Ex.: Londres décide que... pour le gouvernement anglais.

Ex.: Le Quai d'Orsay se refuse à tout commentaire... pour le ministère des Affaires étrangères. Etc. (cf. Métonymie).

METONYMIE Trope qui permet de désigner qqch par le nom d'un autre élément du même ensemble, en vertu d'une relation suffisamment nette.

Ex.: Le phallus dans ce siècle devient doctrinaire. (pour l'instinct sexuel sert aujourd'hui de principe moral.

GRANDILOQUENCE Ton sublime affecté.

Analogues: emphase, pompe, enflure, boursouflure. Adj. Grandiloquent, emphatique, pompeux, enflé, boursouflé, ampoulé, déclamatoire, guindé.

Les anciens distinguaient trois tons dans le discours : le sublime, le tempéré (le soutenu, élevé et noble semble à michemin entre le sublime et le tempéré. La linguistique n'a retenu que trois niveaux de langue, éliminant le sublime au 
profit du soutenu.) et le simple sans parler du bas et du grossier (ils étaient mentionnés mais jugés indignes d'examen).

HYPERBOLE Augmenter ou diminuer excessivement la v érité des

choses pour qu'elle produise plus d'impression. LITTRE. (donc dire plus pour faire entendre moins).

Ex: un bruit à éveiller un mort.

\section{ATTENUATIONS contraire d'hyperbole.}

LITOTE se servir d'une expression qui dit moins pour en faire entendre plus.

Ex: va, je ne te hais point. Corneille, Le Cid.

«Cependant, le régime comportait des vices de fonctionnement qui avaient pu sembler supportables à une époque assez statique, mais qui n'étaient plus compatibles avec les mouvements humains, les changements économiques, les périls extérieurs » 29-33

Une autre technique de persuasion chez de Gaulle, c'est le recours aux extrêmes et aux appositions. Pour lui, l'emploi de ce procédé l'aide à s'exprimer. Il est sûr que son idée est saisie surtout s'il veut inspirer au peuple ce qu'il doit choisir et ce qu'il doit repousser. Ainsi nous avons des mots tels que «se réformer, se briser »(L.1), «s'élever, s'effacer » 
(L.16-18), « au-dedans, au-dehors» (L.17-18), «statique, les mouvements» (L.30-31), «autorité intérieure et assurance extérieure » (L.45), «approuvera ou repoussera ( (L.65), «refleurira ou périra »(L.91), «accepter, refuser » (L.134135

BOURSIN Françoise, Dominique de Villepin et Colin Powell: deux rhétoriques face à face, in Communication et langages, 2005, ${ }^{\circ} 145$, pp.95-106

1 Jacqueline de Romilly, Les grands Sophistes dans l'Athènes de Périclès, "Biblio Essais", Editions de Fallois, Paris, 1988, p.287

C. Peyroutet, Style et rhétorique, Nathan, Paris, 1998, p.108 -110.

${ }^{1}$ Revel, Jean-François, Le style du général, Edition René Julliard, Paris,1959, p.92

DUPRIEZ Bernard, Gradus, Les procédés littéraires, Edition 10/18, Département d'Univers Poche, Paris, 1984, p.387. 